\title{
Mechanistic Evaluation of the Pros and Cons of Digital RT-LAMP for HIV-1 Viral Load Quantification on a Microfluidic Device and Improved Efficiency via a Two-Step Digital Protocol
}

\author{
Bing Sun, ${ }^{\dagger}$ Feng Shen, ${ }^{\ddagger}$ Stephanie E. McCalla, ${ }^{\dagger}$ Jason E. Kreutz, ${ }^{\S}$ Mikhail A. Karymov, ${ }^{\dagger}$
} and Rustem F. Ismagilov*, $\dagger^{\dagger}$

${ }^{\dagger}$ Division of Chemistry and Chemical Engineering, California Institute of Technology, 1200 East California Boulevard, Pasadena, California 91125, United States

\section{Supporting Information}

\begin{abstract}
Here we used a SlipChip microfluidic device to evaluate the performance of digital reverse transcription-loopmediated isothermal amplification (dRT-LAMP) for quantification of HIV viral RNA. Tests are needed for monitoring HIV viral load to control the emergence of drug resistance and to diagnose acute HIV infections. In resource-limited settings, in vitro measurement of HIV viral load in a simple format is especially needed, and single-molecule counting using a digital format could provide a potential solution. We showed here that when one-step dRT-LAMP is used for quantification of HIV RNA, the digital count is lower than expected and is limited by the yield of desired cDNA. We were able to overcome the limitations by developing a microfluidic protocol to manipulate many single molecules in
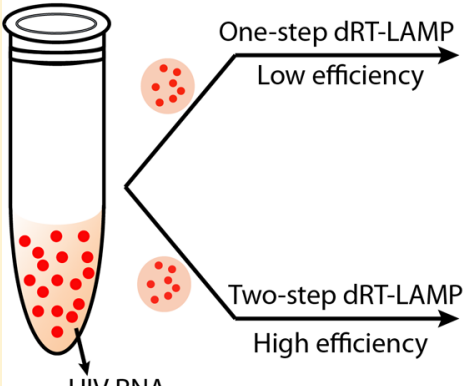

HIV RNA
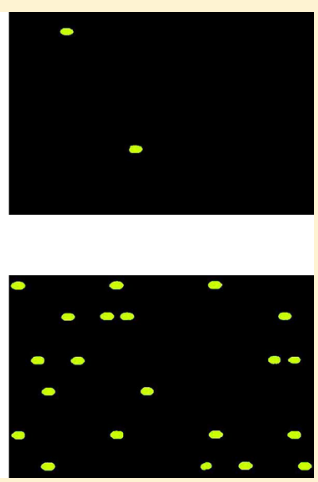
parallel through a two-step digital process. In the first step we compartmentalize the individual RNA molecules (based on Poisson statistics) and perform reverse transcription on each RNA molecule independently to produce DNA. In the second step, we perform the LAMP amplification on all individual DNA molecules in parallel. Using this new protocol, we increased the absolute efficiency (the ratio between the concentration calculated from the actual count and the expected concentration) of dRT-LAMP 10-fold, from 2\% to 23\%, by (i) using a more efficient reverse transcriptase, (ii) introducing RNase $\mathrm{H}$ to break up the DNA:RNA hybrid, and (iii) adding only the BIP primer during the RT step. We also used this two-step method to quantify HIV RNA purified from four patient samples and found that in some cases, the quantification results were highly sensitive to the sequence of the patient's HIV RNA. We learned the following three lessons from this work: (i) digital amplification technologies, including dLAMP and APCR, may give adequate dilution curves and yet have low efficiency, thereby providing quantification values that underestimate the true concentration. Careful validation is essential before a method is considered to provide absolute quantification; (ii) the sensitivity of dLAMP to the sequence of the target nucleic acid necessitates additional validation with patient samples carrying the full spectrum of mutations; (iii) for multistep digital amplification chemistries, such as a combination of reverse transcription with amplification, microfluidic devices may be used to decouple these steps from one another and to perform them under different, individually optimized conditions for improved efficiency.
\end{abstract}

$\mathrm{I}$ $\mathrm{n}$ this paper, we evaluate the potential of using digital reverse transcription loop-mediated isothermal amplification (dRTLAMP) for quantifying the concentration of HIV-1 viral RNA. A simple, convenient quantification method for HIV viral load is important as an in vitro diagnostic tool to aid the 34 million people worldwide with HIV, many of whom live in developing countries and under resource-limited conditions. ${ }^{1}$ As antiretroviral treatment becomes more widely available, viral resistance to first-line antiretroviral drugs is a problem of increasing concern. To combat the spread of drug resistance and make appropriate choices concerning second-line therapy, it is important for patients to undergo regular monitoring of HIV viral load (every 3-4 months) ${ }^{2}$ to check for sharp increases, which indicate the evolution of viral resistance to first-line therapies. Real-time reverse transcription-polymerase chain reaction (RT-PCR) is the most commonly adopted method in reference laboratories, yet it faces multiple challenges in resource-limited conditions because of the cost and technical constraints. It requires dedicated equipment and physical resources such as uninterrupted electricity, air conditioning, refrigeration, an isolated room, and access to clean water. ${ }^{3}$ Moreover, it is a kinetic measurement method and is sensitive to variations in reaction conditions that influence reaction rate. As a result, the reproducibility of this method can be influenced by a variety of perturbations (e.g., fluctuations in

Received: September 20, 2012

Accepted: December 27, 2012

Published: January 16, 2013 
sample quality, reaction time, and temperature), which are potential concerns for HIV viral load quantification, especially in settings where little control over environmental fluctuations is available. ${ }^{4}$

Here we ask whether dRT-LAMP quantification provides an attractive alternative to real-time RT-PCR. In principle, a digital format quantification method could have higher tolerance to variations in reaction rate than real-time bulk measurements because it provides quantitative information via many qualitative yes/no experiments. As is shown in the case of recombinase polymerase amplification (RPA), ${ }^{5}$ a digital format quantification method is robust to temperature variation, because it requires interpreting only end point yes/no results. Moreover, the LAMP reaction can be performed under isothermal conditions and, when calcein is used as an indicator to produce bright fluorescent output, it could allow for easy counting of digital output, either visually or with a portable imaging device such as a cell phone camera instead of a sophisticated fluorescence microscope. This unique property makes this chemistry a viable candidate for resource-limited settings.

In this paper, we used a microfluidic SlipChip ${ }^{6}$ to evaluate the suitability of dRT-LAMP for quantifying HIV viral RNA. It has previously been shown that multiplexed $\mathrm{PCR},{ }^{7} \mathrm{dPCR},{ }^{8}$ dRT-PCR, ${ }^{9}$ and $\mathrm{dRPA}^{5}$ can be performed successfully on SlipChip. In addition, digital LAMP (but not digital RTLAMP) has previously been demonstrated for model doublestranded DNA (dsDNA) targets on other microfluidic devices. $^{10,11}$ However, we found here that performing dRTLAMP in a single step on SlipChip is problematic for quantifying HIV viral RNA: the quantification result using dRT-LAMP was only $2.0 \%$ of that achieved using dRT-PCR with the same template concentration. While bulk RT-LAMP assays for the detection of HIV-1 RNA have been developed, ${ }^{12,13}$ these assays differ from digital assays because the latter is performed at the single-molecule level, which might introduce additional complexity to the reaction. We performed mechanistic studies to understand some of the shortcomings of one-step dRT-LAMP and developed a two-step dRT-LAMP protocol on SlipChip to overcome these limitations. We demonstrated that SlipChip can be used to compartmentalize single molecules and then to implement multistep reactions on those compartments. We further showed that dRT-LAMP can be performed on SlipChip devices made of both glass and plastic, which is a more desirable and cost-effective material for resource-limited settings. Finally, we identified problems, including low overall efficiency and sensitivity to mutations in patient samples, that need to be addressed in the future to make this method suitable for quantitative diagnostics.

\section{EXPERIMENTAL SECTION}

Chemicals and materials used in this paper are described in the Supporting Information. The fabrication, assembly, and loading protocols for glass and plastic SlipChip are based on previous work $^{6,9}$ and are described in the Supporting Information. HIV viral RNA purification from AcroMetrix HIV-1 Panel Copies/ $\mathrm{mL}$ was performed using the iPrep purification instrument. See Supporting Information for more information. Primer sequences for RT-LAMP and RT-PCR amplification and the sequencing results of HIV viral RNA used in the paper are described in the Supporting Information.

ssDNA Synthesis. HIV cDNA was created by reverse transcription of the purified AcroMetrix HIV RNA using the
SuperScript III First-Strand Synthesis SuperMix according to the manufacturer's instructions. Briefly, a mixture of purified HIV RNA (10-fold diluted from the direct elution), $100 \mathrm{nM} \mathrm{B} 3$ primer, 1x Annealing buffer, and water were heated to $65^{\circ} \mathrm{C}$ for $5 \mathrm{~min}$ and then placed on ice for $1 \mathrm{~min}$. A reaction mix and SuperScript III/RNase Out enzyme mix were added to the reaction for a final volume of $40 \mu \mathrm{L}$, and the mixture was placed at $50{ }^{\circ} \mathrm{C}$ for $50 \mathrm{~min}$. The mixture was then heated to $85^{\circ} \mathrm{C}$ for $5 \mathrm{~min}$ to deactivate the reverse transcriptase, chilled on ice, split into $5 \mu \mathrm{L}$ aliquots, and frozen at $-20{ }^{\circ} \mathrm{C}$ until further use. Biotin-labeled DNA was created in a PCR reaction containing a 1:50 dilution of the HIV cDNA, $500 \mathrm{nM}$ biotin-B3 and F3 primers, $500 \mu \mathrm{M}$ dNTPs, $1 \mathrm{U} / \mu \mathrm{L}$ Phusion DNA polymerase, and $1 \mathrm{x}$ of the associated HF buffer mix. After an initial $1 \mathrm{~min}$ enzyme activation step at $98{ }^{\circ} \mathrm{C}$, the reaction was cycled 39 times at $98{ }^{\circ} \mathrm{C}$ for $10 \mathrm{~s}, 58{ }^{\circ} \mathrm{C}$ for $15 \mathrm{~s}$, and $72{ }^{\circ} \mathrm{C}$ for $15 \mathrm{~s}$ and finished with a $5 \mathrm{~min}$ polishing step at $72{ }^{\circ} \mathrm{C}$. The resulting DNA product was analyzed on a $1.2 \%$ agarose gel in TBE buffer stained with $0.5 \mu \mathrm{g} / \mathrm{mL}$ ethidium bromide. The specific band was cut out and purified using the Wizard SV gel and PCR cleanup kit according to the manufacturer's instructions and eluted into $50 \mu \mathrm{L}$ of nuclease-free water. A $50 \mu \mathrm{L}$ amount of streptavidin MyOne T1 magnetic beads were primed by slowtilt rotation for $24 \mathrm{~h}$ in $20 \mathrm{mM} \mathrm{NaOH} .{ }^{14}$ The beads were washed one time with water and four times with binding buffer (5 mM Tris, $0.5 \mathrm{mM}$ EDTA, $1 \mathrm{M} \mathrm{NaCl}, 0.05 \%$ Tween-20) and resuspended in $30 \mu \mathrm{L}$ of $2 \mathrm{x}$ concentrated binding buffer. Thirty microliters of PCR product was added to the beads and incubated for $15 \mathrm{~min}$ while gently rotating to allow binding of the DNA to the magnetic beads. The beads were separated with a magnet, the supernatant was removed, and the beads were resuspended in $40 \mu \mathrm{L}$ of $20 \mathrm{mM} \mathrm{NaOH}$ and incubated for 10 min on a rotator to separate the nonbiotinylated strand. The beads were then separated with a magnet, and the supernatant containing the ssDNA was collected and mixed with $20 \mu \mathrm{L}$ of $40 \mathrm{mM} \mathrm{HCl}$. The resulting ssDNA was then purified using an ssDNA/RNA cleaner and concentrator kit, eluted in $20 \mu \mathrm{L}$ of water, and analyzed on an Agilent RNA nano bioanalyzer to confirm the size and integrity of the final product.

dRT-LAMP and dLAMP Amplification on SlipChip. To amplify HIV viral RNA using the one-step RT-LAMP method, the RT-LAMP mix contained the following: $20 \mu \mathrm{L}$ of RM, $2 \mu \mathrm{L}$ of BSA $(20 \mathrm{mg} / \mathrm{mL}), 2 \mu \mathrm{L}$ of EM, $2 \mu \mathrm{L}$ of $\mathrm{FD}, 2 \mu \mathrm{L}$ of primer mixture $(20 \mu \mathrm{M}$ BIP/FIP, $10 \mu \mathrm{M}$ LooP_B/Loop_F, and 2.5 $\mu \mathrm{M} \mathrm{B} 3 / \mathrm{F} 3)$, various amounts of template solution, and enough nuclease-free water to bring the volume to $40 \mu \mathrm{L}$. The solution was loaded onto a SlipChip and heated at $63{ }^{\circ} \mathrm{C}$ for $60 \mathrm{~min}$.

To amplify HIV viral RNA using the two-step RT-LAMP method, the first solution contained the following: $10 \mu \mathrm{L} \mathrm{RM}, 1$ $\mu \mathrm{L}$ of BSA, $0.5 \mu \mathrm{L}$ of EXPRESS SYBR GreenER RT module (part of EXPRESS One-Step SYBR GreenER Universal), 0.5 $\mu \mathrm{L}$ of BIP primer $(10 \mu \mathrm{M})$, various amounts of template solution, and enough nuclease-free water to bring the volume to $20 \mu \mathrm{L}$. The second solution contained: $10 \mu \mathrm{L}$ of $\mathrm{RM}, 1 \mu \mathrm{L}$ of BSA, $2 \mu \mathrm{L}$ of DNA polymerase solution (from LoopAmp DNA amplification kit), 1 or $2 \mu \mathrm{L}$ of $\mathrm{FD}, 2 \mu \mathrm{L}$ of other primer mixture $(20 \mu \mathrm{M}$ FIP, $17.5 \mu \mathrm{M}$ FIP, $10 \mu \mathrm{M}$ LooP_B/Loop_F, and $2.5 \mu \mathrm{M}$ F3), $1 \mu \mathrm{L}$ of Hybridase Thermostable RNase $\bar{H}$, and enough nuclease-free water to bring the volume to $20 \mu \mathrm{L}$. The first solution was loaded onto a SlipChip device and incubated at 37 or $50{ }^{\circ} \mathrm{C}$, the second solution was loaded onto the same device and mixed with the first solution, and the entire device was incubated at $63{ }^{\circ} \mathrm{C}$ for $60 \mathrm{~min}$. 
A

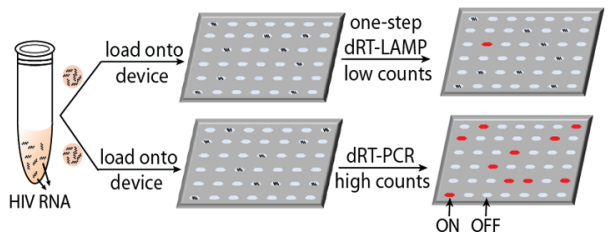

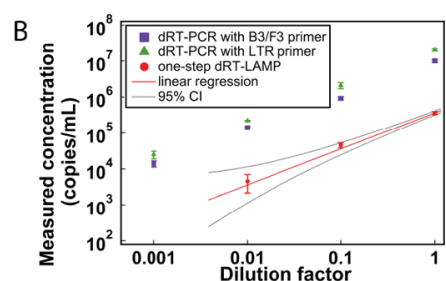

Figure 1. One-step RT-LAMP has lower efficiency than RT-PCR in digital format when quantifying HIV RNA on SlipChip. (A) Schematic demonstration of one-step dRT-LAMP and dRT-PCR in which use of the same template concentration results in different digital counts. (B) Quantification results of HIV viral RNA (copies/mL) in a serial dilution experiment on SlipChip using one-step dRT-LAMP $(n=4)$, dRT-PCR with B3/F3 primers $(n=3)$, and dRT-PCR with LTR primers $(n=3)$. Error bars represent standard deviation.

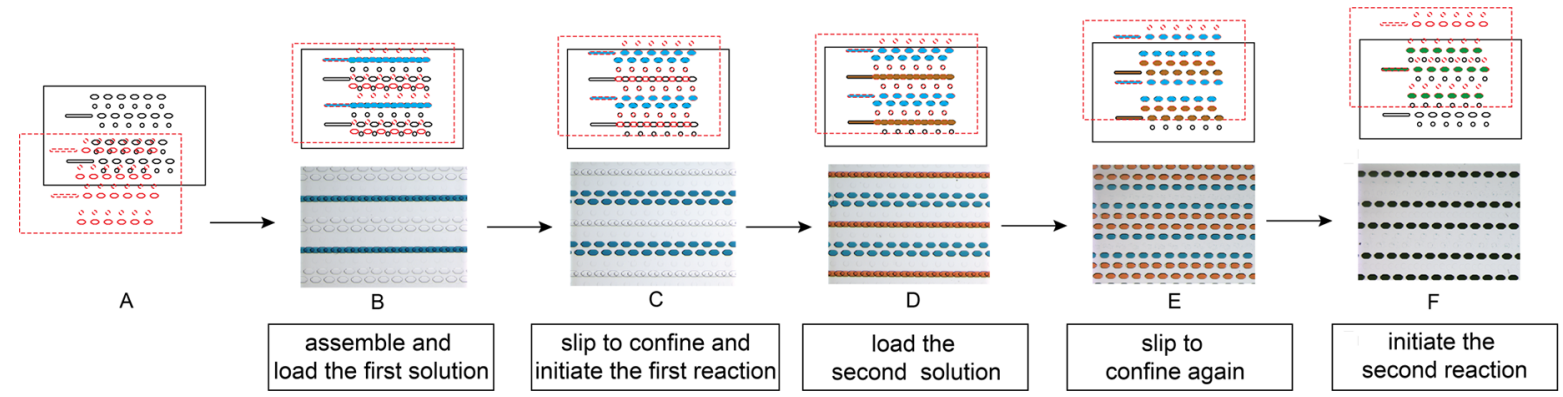

Figure 2. Schematic drawings and images showing the operation of SlipChip for two-step dRT-LAMP. (A) Top (black) and bottom (red) plates of the SlipChip before assembly. (B) Assembled SlipChip after loading of RT solution. (C) RT solution containing RNA molecules confined to individual wells after slipping. (D) Loading of LAMP reagent mixture after RT reaction has completed. (E) LAMP reagent mixture confined to individual wells after slipping again. (F) Reaction initiated after slipping to mix RT and LAMP wells.

To amplify $\lambda$-DNA, the LAMP mix contained the following: $20 \mu \mathrm{L}$ of $\mathrm{RM}, 2 \mu \mathrm{L}$ of BSA $(20 \mathrm{mg} / \mathrm{mL}), 2 \mu \mathrm{L}$ of DNA polymerase, $2 \mu \mathrm{L}$ of $\mathrm{FD}, 2 \mu \mathrm{L}$ of primer mixture $(20 \mu \mathrm{M} \mathrm{BIP} /$ FIP, $10 \mu \mathrm{M}$ LooP_B/Loop_F and $2.5 \mu \mathrm{M}$ B3/F3), various amounts of template solution, and enough nuclease-free water to bring the volume to $40 \mu \mathrm{L}$. The same loading protocol as above was performed, and the device was incubated at $63{ }^{\circ} \mathrm{C}$ for $70 \mathrm{~min}$.

To amplify ssDNA, the LAMP mix contained the following: $20 \mu \mathrm{L}$ of RM, $2 \mu \mathrm{L}$ of BSA, $2 \mu \mathrm{L}$ of DNA polymerase, $2 \mu \mathrm{L}$ of FD, $2 \mu \mathrm{L}$ of primer mixture $(20 \mu \mathrm{M}$ BIP/FIP, $10 \mu \mathrm{M}$ LooP_B/ Loop_F, and $2.5 \mu \mathrm{M} \mathrm{B} 3 / \mathrm{F} 3)$, various amounts of template solution, and enough nuclease-free water to bring the volume to $40 \mu \mathrm{L}$. The same loading protocol as above was performed, and the device was incubated at $63{ }^{\circ} \mathrm{C}$ for $60 \mathrm{~min}$.

dRT-PCR Amplification on SlipChip. To amplify HIV viral RNA, the RT-PCR mix contained the following: $20 \mu \mathrm{L}$ of $2 \mathrm{X}$ Evagreen SuperMix, $2 \mu \mathrm{L}$ of BSA, $1 \mu \mathrm{L}$ of EXPRESS SYBR GreenER RT module, $1 \mu \mathrm{L}$ of each primer $(10 \mu \mathrm{M}), 2 \mu \mathrm{L}$ of template, and enough nuclease-free water to bring the volume to $40 \mu \mathrm{L}$. The amplification was performed under the same conditions as reported previously ${ }^{9}$ except for a shortened reverse transcription step of $10 \mathrm{~min}$. Image acquisition and analysis methods are described in the Supporting Information.

\section{RESULTS AND DISCUSSION}

We first compared quantification results of HIV viral RNA purified from Acrometrix panel at four dilutions using two different digital chemistries: dRT-PCR and dRT-LAMP. dRTPCR with primers targeting the LTR region of HIV was used as the standard because it has been validated previously. ${ }^{9}$ B3/F3 primers taken from the LAMP primer set were also used in dRT-PCR for comparison. For RT-LAMP, we used a commercial kit with published primers targeting the p24 gene. $^{12}$ The priming region of HIV RNA was sequenced to confirm that there was no mismatch between primers and template (see Supporting Information). HIV viral RNA concentration was calculated based on the number of observed positive wells ("digital counts") on a single device according to the Poisson statistical analysis method discussed in a previous paper. ${ }^{15}$ The overall result was surprising: while dilution curves with good linearity were obtained for both digital chemistries listed above, the absolute values were dramatically different. For the two chemistries, the same concentration of RNA gave quantification values that differed by $\sim 50$-fold, with dRTLAMP giving the lowest digital counts (Figure 1). The quantification values calculated from digital counts for onestep dRT-LAMP were on average only $2.0 \%$ of the values for the dRT-PCR standard with LTR primers and were on average $3.9 \%$ of the values for dRT-PCR with B3/F3 primers. One could interpret these results optimistically and state that as long as one has a calibration curve, one could perform adequate quantification no matter the efficiency of the particular digital amplification chemistry. However, there are also two alternative interpretations, admittedly more pessimistic in nature. First, seeing a proper dilution curve does not mean that the quantification is correct: digital "absolute" quantification is not absolute unless rigorously validated by independent experiments. Second, using an inefficient digital method like the one-step dRT-LAMP shown in Figure 1 is inadequate for quantifying lower concentrations of analytes. This is a problem, especially in the case of HIV viral load, where changes most often in the range of 50 to 5000 copies of RNA per mL need to be quantified to make clinical decisions. ${ }^{16}$ Therefore, we acted on the more pessimistic interpretation of these results and worked on understanding why the digital efficiency of dRTLAMP is so low, toward the goal of improving its efficiency.

Given the poor performance of one-step dRT-LAMP, we performed a parallel control experiment on the same SlipChip using a published and well-characterized LAMP system based 

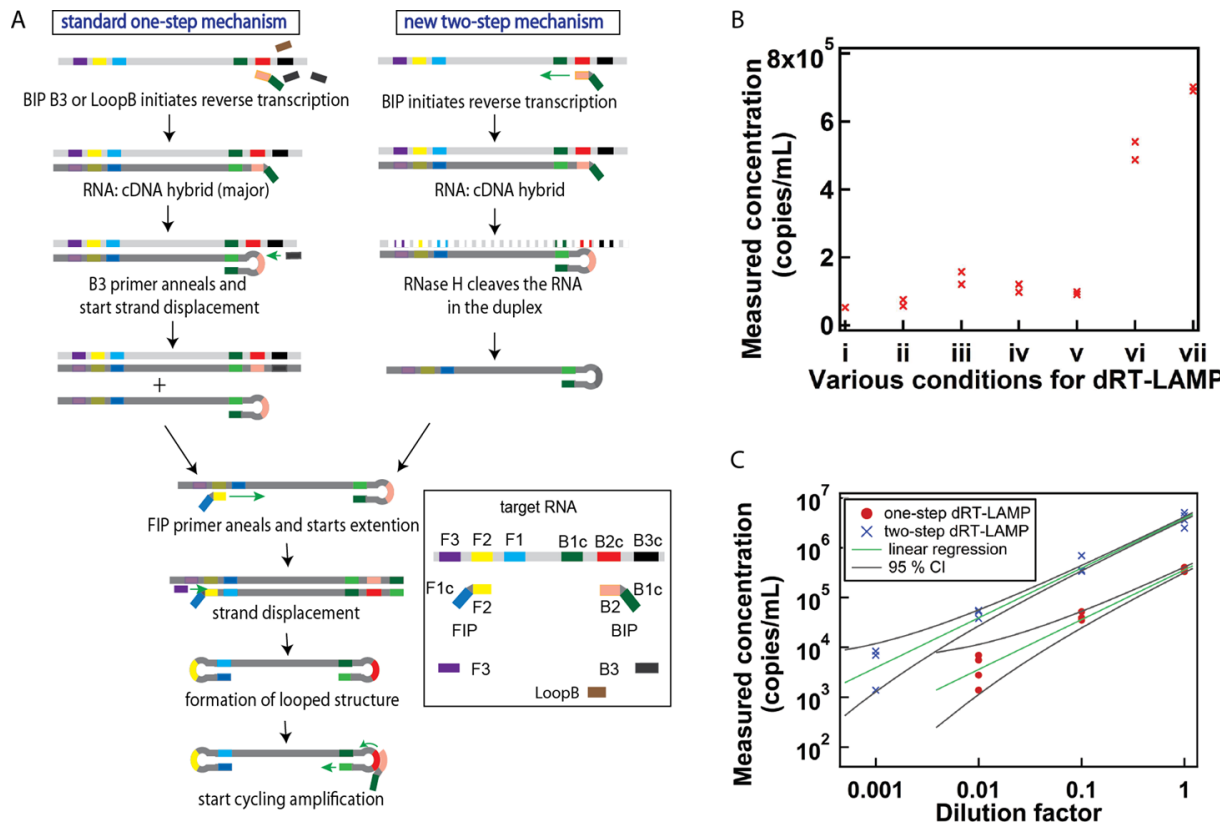

Figure 3. A modified two-step mechanism for RT-LAMP leads to higher efficiency compared with a one-step protocol in digital format. (A) Comparison of the new two-step RT-LAMP mechanism versus the published one-step RT-LAMP mechanism. In the initial step, the two-step mechanism uses only BIP primer, and the one-step protocol uses all primers together. After the formation of the DNA:RNA hybrid, the two-step mechanism uses RNase $\mathrm{H}$ instead of B3 primer-initiated strand displacement to release the cDNA. The two mechanisms converge after the formation of first-strand cDNA that is looped on one side. Figure truncated; details of cycling amplification including the loop primers' annealing are not shown here and can be found in previous papers. ${ }^{20,21}$ Inset shows designated primers: B2 and B2c, B3 and B3c, F1 and F1c are complementary sequences, respectively. (B) Concentration of HIV viral RNA (copies/mL) measured with dRT-LAMP using different protocols and the same template concentration. (i) one-step dRT-LAMP; (ii) two-step dRT-LAMP, all primers in RT step, AMV RT; (iii) two-step, BIP in RT step, AMV RT; (iv) two-step, BIP in RT step, Superscript III; (v) two-step, BIP in RT step, AMV RT, with RNase H; (vi) two-step, BIP in RT step, Superscript III, with RNase H; (vii) two-step, BIP in RT step, Superscript III, with RNase H, 0.5x calcein $(n=2)$. (C) Quantification results of HIV viral RNA (copies $/ \mathrm{mL})$ in a serial dilution experiment on SlipChip using one-step dRT-LAMP and two-step dRT-LAMP $(n=4)$. Error bars represent standard deviation.

on $\lambda$-DNA ${ }^{10,17}$ to confirm that the SlipChip device did not introduce any artifacts when used with LAMP chemistry. The concentration of the $\lambda$-DNA stock solution was measured with a Qubit fluorometer and used as the expected original concentration for further dilution. dLAMP was performed according to a previous publication. ${ }^{10}$ Without a preheating step at $95{ }^{\circ} \mathrm{C}$ to dissociate double-stranded DNA template, we observed zero digital counts. Unfortunately, previous reports on dLAMP with $\lambda$-DNA did not specify whether preheating was or was not required. ${ }^{10,16}$ Neither was this preheating step specified in a recent paper where $\beta$-actin DNA was used as the template for dLAMP reaction. ${ }^{11}$ When a preheating step was introduced, the quantification value of dLAMP on SlipChip increased to $62 \%$ of the expected value (Figure S2). The results depend on the details of how preheating is performed and how quickly DNA is cooled and used in the subsequent amplification step. ${ }^{18,19}$ We did not optimize the preheating protocol because it is not required by single-stranded HIV RNA (aside from the secondary structure), so we did not expect a perfectly efficient quantification. It was concluded that the glass SlipChip platform was not introducing artifacts significant enough to be responsible for the $2.0 \%$ efficiency observed for one-step dRT-LAMP.

To determine the cause of the lower efficiency of one-step dRT-LAMP compared to dRT-PCR, we attempted to decouple the reverse transcription step from the amplification step and look at the efficiency of each step independently. We designed a glass SlipChip device for performing dRT-LAMP in two steps (Figure 2). The device was composed of two glass plates with wells and channels etched on their facing sides. The plates of the chip were assembled and aligned to allow for the loading, compartmentalization, incubation, and mixing of reagents in multiple steps. This chip is reminiscent of, but not the same as, the chip previously described for digital RPA. ${ }^{5}$ First, a buffered solution containing template, primer, and RT enzyme was loaded into wells on the chip (Figure 2B). Next, the plates of the chip were slipped relative to one another to confine single HIV viral RNA molecules into droplets (Figure 2C). A first incubation step was performed here to allow for reverse transcription. cDNA was synthesized from RNA in each compartment during the reverse transcription step. Then, a second solution containing the LAMP reagent mixture and other primers was loaded (Figure 2D) and split into compartments by slipping (Figure $2 \mathrm{E}$ ). Finally, each of the compartments containing a cDNA molecule was combined with a compartment containing LAMP reagents, and the entire device was incubated at a higher temperature for amplification. The fluorescence microphotograph of part of a loaded glass device after amplification, along with a line scan showing intensity, is presented in the Supporting Information.

We then studied the influence of several factors, including the orders of primers annealing to the RNA template, the effect of different reverse transcriptases, and the ease of breaking up the DNA:RNA hybrid independently, and proposed a new twostep mechanism (Figure 3).

In the RT-LAMP mechanism, a BIP primer should first hybridize to target RNA to start the synthesis of the first-strand cDNA, which will be released from the RNA later by strand 
displacement initiated by a B3 primer. $^{20,21}$ However, we hypothesized that if instead of BIP primer, Loop_B primer or B3 primer hybridizes to the target RNA first and starts reverse transcription, no looped structure on the $3^{\prime}$ size of $\mathrm{cDNA}$ will be formed after strand displacement; once B3 primer hybridizes to RNA and forms an RNA:DNA duplex, it is difficult for other primers to insert into the duplex and start reverse transcription because of the high stability of RNA:DNA. ${ }^{22}$ As a result, we hypothesized that it is critical to start reverse transcription with BIP primer to make sure each RNA molecule is amplified. We tested this hypothesis by performing a two-step protocol and using different primer compositions in the RT step: one has all LAMP primers and the other has only BIP primer (Figure 3A). With all primers, $3.2 \%$ efficiency relative to dRT-PCR using LTR primers was observed, while with only BIP primer, the efficiency of dRT-LAMP increased about 2-fold to $6.8 \%$ (Figure $3 \mathrm{~B}(\mathrm{ii}$ and iii)). All of the dRT-LAMP experiments reported in Figure $3 \mathrm{~B}$ were performed using an HIV RNA solution of the same concentration as the 0.1 dilution in Figure 1B. Given these results, we concluded that the order in which the primers anneal to the target influences the initiation efficiency of the reaction.

Different reverse transcriptases are reported to have different efficiencies in first-strand cDNA synthesis. ${ }^{23}$ In bulk experiments where multiple copies of the target are initially present, a high RT efficiency is not crucial for the reaction to proceed because the LAMP reaction is so sensitive that even with a very low RT yield, the synthesized cDNA molecules can still initiate subsequent amplification. This could also explain why RTLAMP can be performed at a temperature $\left(63^{\circ} \mathrm{C}\right)$ beyond the optimal temperature of AMV reverse transcriptase $\left(37^{\circ} \mathrm{C}\right.$ to 42 $\left.{ }^{\circ} \mathrm{C}\right)$. However, at the single-molecule level, to get an accurate absolute digital count of RNA molecules, it is necessary to choose a reverse transcriptase with a high RT yield to obtain cDNA from as many RNA molecules as possible (and ideally, from each molecule) in the reverse transcription step. We replaced AMV reverse transcriptase with Superscript III reverse transcriptase, which is reported to have a higher reverse transcription efficiency. ${ }^{23}$ However, we did not observe any efficiency increase (Figure 3B(iii and iv)). Considering the fact that Superscript III (similar to Superscript II) has low strand displacement activity ${ }^{24}$ due to the reduced RNase $\mathrm{H}$ activity, while AMV reverse transcriptase has strong strand displacement activity, and that the release of cDNA from the RNA:cDNA hybrid relies on the strand displacement initiated by $\mathrm{B} 3$ primer, we hypothesized that even if more cDNA is synthesized by Superscript III, it may not be released from the hybrid. To test this hypothesis, we added $\mathrm{RNase} H$, which can cleave RNA from an RNA:DNA duplex through a hydrolytic mechanism, to aid the release of the cDNA (Figure 3A). However, because the presence of $\mathrm{RNase} \mathrm{H}$ will interfere with the activity of reverse transcriptase, it can only be added after the reverse transcription is complete. We used the two-step protocol and added a thermally stable $\mathrm{RNase} \mathrm{H}$ to the second reaction mixture. To make sure we are looking at only the effect of RNase $\mathrm{H}$ breaking up the RNA:DNA hybrid, without the interference from any strand displacement, we removed the B3 primer from the second reaction mixture because it was no longer needed. Both reverse transcriptases were tested with $\mathrm{RNase} H$ in this protocol. We observed no improvement using RNase $\mathrm{H}$ together with AMV reverse transcriptase (Figure 4A(v)), confirming that AMV strand displacement activity was sufficient for the release of the first strand ssDNA. However,
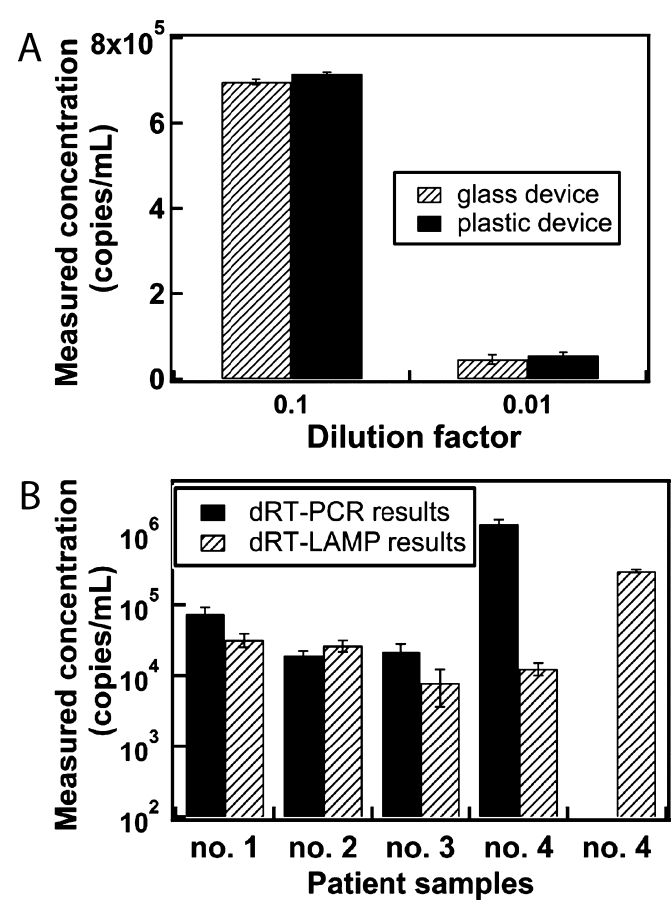

Figure 4. (A) Quantification results of HIV viral RNA (copies $/ \mathrm{mL}$ ) on a plastic SlipChip at two dilution factors, with comparisons to results obtained on a glass device $(n=3)$. (B) Quantification of HIV viral RNA purified from patient samples using dRT-LAMP and dRTPCR. For sample no. 4, quantification results using dRT-LAMP with primers corrected for mutations are shown in the rightmost column of the figure $(n=3)$. Error bars represent standard deviation.

under the same conditions, using RNase $\mathrm{H}$ together with Superscript III significantly increased the quantification results of dRT-LAMP from $5.3 \%$ to $25 \%$ (Figure 3B (iv and vi)) relative to that quantified by dRT-PCR with LTR primers.

In a LAMP reaction, calcein is used as the readout fluorophore. ${ }^{25}$ Yet because calcein is a good ligand for metal ions, it might interfere with enzyme activities or dsDNA stability. We tested the effect of adding different amounts of calcein and found that decreasing the calcein to half $(0.5 \times)$ of the recommended concentration gave the highest efficiency (Figure $3 \mathrm{~B}($ vii)). We also performed two-step dRT-LAMP in the temperature range of $57{ }^{\circ} \mathrm{C}$ to $66^{\circ} \mathrm{C}$ and determined the optimal temperature to be $63{ }^{\circ} \mathrm{C}$ (data not shown).

The assay was reproducible in our hand, and we believe that $n=2$ in each reaction condition is sufficient for the purpose of optimizing the reaction protocols. However, to confirm the robustness of this protocol, we performed two-step dRT-LAMP with the optimized protocol at four different concentrations in quadruplicate and compared the results to one-step dRTLAMP (Figure 3C). The quantification results showed a linear relationship with the dilution level for both protocols. The $P$ value of the linear regression for one-step dRT-LAMP is $4.0 \times$ $10^{-11}$ and for two-step dRT-LAMP is $7.4 \times 10^{-9}$, both of which are $\ll 0.01$. Therefore, we rejected the null hypothesis that there is no relationship between the data points that composed each curve. The average efficiency compared to dRT-PCR with LTR primers was $2.0 \% \pm 0.2 \%$ for one-step dRT-LAMP, and $23.2 \% \pm 2 \%$ for two-step dRT-LAMP; thus, it increased by about 10 -fold.

Next, we excluded the influence of the RT step and assessed the efficiency of the LAMP step independently by performing 
dLAMP using a 225 nucleotide (nt) ssDNA template of the same sequence as the p24 LAMP priming region. We hypothesized that if the formation of the desired singlestranded cDNA is no longer the bottleneck in the two-step protocol, then ALAMP using ssDNA as template would have similar efficiency to two-step dRT-LAMP using HIV viral RNA as template. Because the $225 \mathrm{nt}$ ssDNA did not include the LTR region, we could not use dRT-PCR with LTR primers as a reference with which to compare. Instead, we used dRT-PCR with B3/F3 primers as a reference. We have shown that twostep dRT-LAMP gave on average 39\% efficiency compared with dRT-PCR using B3/F3 primers in the serial dilution experiments (Figure 1). Here, we observed that dLAMP using synthesized ssDNA as template gave $33 \%$ efficiency compared to $\mathrm{dPCR}$ with $\mathrm{B} 3 / \mathrm{F} 3$ primers using ssDNA as template. We assumed that dRT-PCR and APCR should have the same efficiency because the HIV RNA template and the ssDNA template had the same sequence (see Supporting Information), and the same pair of primers was used to amplify them. Then, we concluded that two-step IRT-LAMP and dLAMP had comparable efficiency. This result indicated that by using the two-step protocol, we have solved the problem of forming the desired single-stranded cDNA.

To evaluate the robustness of this dRT-LAMP chemistry in resource-limited settings, we also tested its compatibility with plastic material and its tolerance to sequence divergence of patient samples. We performed two-step dRT-LAMP of HIV viral RNA on a plastic SlipChip device with the same design as the glass device used in the previous experiments. The RNA quantification results using two-step dRT-LAMP on the plastic SlipChip device and the glass SlipChip device agreed well with each other (Figure 4A). This result indicated that the quantification results are not sensitive to the details of surface chemistry of the SlipChip, as we expected, because the surface chemistry control occurs at the liquid-liquid interface of the aqueous solution and the lubricating fluid. ${ }^{6,7,26}$ This result also confirmed the possibility of using a potentially low-cost plastic device as the platform for performing dRT-LAMP.

We also tested the performance of two-step dRT-LAMP using HIV viral RNA purified from patient samples and compared these results to measurements from dRT-PCR. Plasma samples from four different patients were purified using a Roche TNAI kit. Both two-step dRT-LAMP with $\mathrm{p} 24$ primers and dRT-PCR with LTR primers were performed to quantify the RNA concentration. The dRT-LAMP quantification results were $43 \% \pm 9 \%, 139 \% \pm 33 \%, 37 \% \pm 20 \%$, and $0.9 \% \pm 0.2 \%$ relative to the corresponding $\mathrm{dRT}$-PCR results, respectively (Figure 4B). The p24 region of the purified HIV viral RNA was sequenced (sequences listed in the Supporting Information). There were $3,2,5$, and 5 point mutations in the priming regions of sample nos. 1, 2, 3, and 4, respectively. Therefore, the general conclusion is that the efficiency of dRT-LAMP is sensitive to mutations in the target sequence, with more mutations leading to lower efficiency. In particular, for sample no. 4 , according to the sequencing results, two of the mutations are at the $3^{\prime}$ end of the BIP priming region and are adjacent to each other, which we hypothesized dramatically decreased the efficiency of reaction. To test this hypothesis, we performed two-step dRT-LAMP on sample no. 4 with BIP primer corrected at these two positions and its efficiency increased from $0.9 \% \pm 0.2 \%$ to $22 \% \pm 1 \%$. Because a LAMP reaction requires three pairs of primers to proceed, its specificity is higher than that of PCR, which requires only one pair of primers. However, we speculate that this high specificity may also make the dLAMP method more sensitive to mutations in the priming regions. It would be valuable to take advantage of the high specificity of this method while maintaining good tolerance to mutations in real patient samples. To achieve this goal, more than one set of primers could be included in a single assay to ensure a higher probability of accurate readings, although this idea needs to be tested experimentally.

\section{CONCLUSION}

We demonstrated in this paper that one-step dRT-LAMP for HIV viral RNA showed only $2.0 \%$ efficiency relative to that observed using dRT-PCR. In particular, we observed that the efficiency of dRT-LAMP for HIV viral RNA quantification is limited by the efficiency of reverse transcription, the order of primer annealing, and the efficiency of the breakup of DNA:RNA duplexes. We solved these problems by using a two-step approach that enabled us to use a more efficient reverse transcriptase, to add only the BIP primer during the RT step, and to introduce RNase $\mathrm{H}$ to break up the DNA:RNA hybrid. Technologies that enable multistep manipulation of many volumes in parallel, such as SlipChip, provide a platform for this multistep protocol for dRT-LAMP. Such microfluidic technologies are useful for mechanistic studies of dLAMP and other digital, single-molecule reactions. Furthermore, the technologies that can be made simple enough for use in resource-limited settings would be useful for deploying digital single-molecule amplification for diagnostics applications.

Single-molecule counting using dLAMP and dRT-LAMP is attractive because it is isothermal and therefore does not require thermocycling equipment, is compatible with plastics, and provides a bright signal from the calcein detection system that should be readable by a cell phone. Although the efficiency of dRT-LAMP for quantification of HIV RNA has been improved over 10-fold, it ideally should be further improved by about 4-fold. A more significant problem is the sensitivity of digital amplification to mutations in the target regions. This problem should be especially severe for viruses such as HIV that mutate rapidly, and for other targets, the severity of this problem remains to be established. General solutions to this problem for dLAMP and dRT-LAMP protocols are needed. Furthermore, comparisons of the sensitivity of different digital amplification methods and their sensitivity to mutations found in a broad cohort of patient samples are also needed. Digital amplification technologies should not be assumed to provide absolute quantification until validated against an absolute standard and, for diagnostic applications, until validated on a representative set of patient samples. Should all of these issues be resolved, we are optimistic that digital single molecule technologies would become useful for quantifying molecules under resource-limited settings.

\section{ASSOCIATED CONTENT}

\section{Supporting Information}

Additional information as noted in text. This material is available free of charge via the Internet at http://pubs.acs.org.

\section{AUTHOR INFORMATION}

\section{Corresponding Author}

*E-mail: rustem.admin@caltech.edu.

Present Addresses

${ }^{\ddagger}$ SlipChip, LLC, 129 N. Hill Ave., Pasadena, CA 91106. 
${ }^{\S}$ Department of Chemistry, University of Washington, Seattle, WA 98195

\section{Author Contributions}

B.S., F.S., S.E.M., and R.F.I. designed the SlipChip experiments. B.S. performed experiments and data analysis. S.E.M. performed ssDNA synthesis. B.S., S.E.M., and R.F.I. wrote the paper. J.E.K. contributed to the design of the two-step SlipChip and data analysis. M.A.K. provided suggestions on experiment design.

\section{Notes}

The authors declare the following competing financial interest(s): Disclosure: Feng Shen and Rustem F. Ismagilov have a financial interest in SlipChip LLC.

\section{ACKNOWLEDGMENTS}

This work was supported by NIH Grant R01EB012946 administered by the National Institute of Biomedical Imaging and Bioengineering and by the NIH Director's Pioneer Award program, part of the NIH Roadmap for Medical Research (5DP1OD003584). We thank Bridget Samuels for contributions to writing and editing this manuscript. We thank Dr. Loren Joseph and Dr. Reddy Poluru for providing RNA purified from patient samples.

\section{REFERENCES}

(1) WHO/UNAIDS/UNICEF. Global HIV/AIDS response: epidemic update and health sector progress towards universal access: progress report 2011, World Health Organization, 2011. http://www.who.int/hiv/ pub/progress_report2011/summary_en.pdf.

(2) Panel on Antiretroviral Guidelines for Adults and Adolescents. Guidelines for the use of antiretroverial agents in HIV-1-infected adults and adolescents, Department of Health and Human Services, 2012. http:// www.aidsinfo.nih.gov/contentfiles/lvguidelines/adultandadolescentgl. pdf. Accessed on 09/15/2012.

(3) Fiscus, S. A.; Cheng, B.; Crowe, S. M.; Demeter, L.; Jennings, C.; Miller, V.; Respess, R.; Stevens, W. PLoS Med. 2006, 3, e417 (the Forum for Collaborative HIV Research Alternative Viral Load Assay Working Group, accessed 8/28/2012).

(4) Murphy, J.; Bustin, S. A. Expert Rev. Mol. Diagn. 2009, 9, 187.

(5) Shen, F.; Davydova, E. K.; Du, W.; Kreutz, J. E.; Piepenburg, O.; Ismagilov, R. F. Anal. Chem. 2011, 83, 3533.

(6) Du, W.; Li, L.; Nichols, K. P.; Ismagilov, R. F. Lab Chip 2009, 9, 2286

(7) Shen, F.; Du, W.; Davydova, E. K.; Karymov, M. A.; Pandey, J.; Ismagilov, R. F. Anal. Chem. 2010, 82, 4606.

(8) Shen, F.; Du, W.; Kreutz, J. E.; Fok, A.; Ismagilov, R. F. Lab Chip 2010, 10, 2666.

(9) Shen, F.; Sun, B.; Kreutz, J. E.; Davydova, E. K.; Du, W.; Reddy, P. L.; Joseph, L. J.; Ismagilov, R. F. J. Am. Chem. Soc. 2011, 133, 17705.

(10) Gansen, A.; Herrick, A. M.; Dimov, I. K.; Lee, L. P.; Chiu, D. T. Lab Chip 2012, 12, 2247.

(11) Zhu, Q.; Mu, Y. Lab Chip 2012, 12, 4755.

(12) Curtis, K. A.; Rudolph, D. L.; Owen, S. M. J. Virol. Methods 2008, 151, 264

(13) Hosaka, N.; Ndembi, N.; Ishizaki, A.; Kageyama, S.; Numazaki,

K.; Ichimura, H. J. Virol. Methods 2009, 157, 195.

(14) Wilson, R. Nucleic Acid Ther. 2011, 21, 437.

(15) Kreutz, J. E.; Munson, T.; Huynh, T.; Shen, F.; Du, W.; Ismagilov, R. F. Anal. Chem. 2011, 83, 8158.

(16) Calmy, A.; Ford, N.; Hirschel, B.; Reynolds, S. J.; Lynen, L.; Goemaere, E.; de la Vega, F. G.; Perrin, L.; Rodriguez, W. Clin. Infect. Dis. 2007, 44, 128.

(17) Goto, M.; Honda, E.; Ogura, A.; Nomoto, A.; Hanaki, K. I. BioTechniques 2009, 46, 6.

(18) Suzuki, R.; Ihira, M.; Enomoto, Y.; Yano, H.; Maruyama, F.; Emi, N.; Asano, Y.; Yoshikawa, T. Microbiol. Immunol. 2010, 54, 466.
(19) Geojith, G.; Dhanasekaran, S.; Chandran, S. P.; Kenneth, J. J. Microbiol. Methods 2011, 84, 71.

(20) Notomi, T.; Okayama, H.; Masubuchi, H.; Yonekawa, T.; Watanabe, K.; Amino, N.; Hase, T. Nucleic Acids Res. 2000, 28, e63.

(21) Nagamine, K.; Hase, T.; Notomi, T. Mol. Cell. Probes 2002, 16, 223.

(22) Barone, F.; Cellai, L.; Matzeu, M.; Mazzei, F.; Pedone, F. Biophys. Chem. 2000, 86, 37.

(23) Ståhlberg, A.; Kubista, M.; Pfaffl, M. Clin. Chem. 2004, 50, 1678.

(24) Kelleher, C. D.; Champoux, J. J. J. Biol. Chem. 1998, 273, 9976.

(25) Tomita, N.; Mori, Y.; Kanda, H.; Notomi, T. Nat. Protocols 2008, 3, 877.

(26) Li, L.; Du, W.; Ismagilov, R. J. Am. Chem. Soc. 2009, 132, 106. 